\title{
UROGENITAL TRAUMA
}

\section{Straddle injuries to the bulbar urethra: management and outcomes in 78 patients} Park S, McAninch JW

Department of Urology, University of California School of Medicine and Urology Service, San Francisco General Hospital, USA

J Urol. 2004; 171(2 Pt 1): 722-5

Purpose: We describe our experience with blunt straddle injuries to the anterior urethra and identify factors that may affect patient outcome.

Materials and Methods: We reviewed the San Francisco General Hospital Urologic Trauma data base to identify men with blunt straddle injury. We analyzed presentation and initial management, location and length of urethral stricture, surgical options, and long-term outcome after reconstruction.

Results: Of 78 patients, $40 \%$ presented to the emergency department acutely and $60 \%$ presented 6 months to 10 years after injury complaining of obstructive symptoms, of whom $30 \%$ reported at least 1 episode of urinary retention. Initial acute management was suprapubic cystostomy in $81 \%$ of cases and primary realignment in $19 \%$. Urethral strictures were predominantly located in the proximal bulb. Mean stricture length was significantly longer in men with delayed presentation $(2.7 \mathrm{vs} 1.8 \mathrm{~cm}, \mathrm{p}<0.05)$. No relationship was found between stricture length and the mechanism of injury or initial management technique. However, patients who had undergone primary realignment required complex flap or graft urethroplasty at a greater rate compared with men who had undergone suprapubic diversion $(p=0.054)$. Transperineal urethroplasty was required in $92 \%$ of patients with the majority undergoing end-to-end anastomosis. The success rate was $95 \%$ at a mean followup of 25 months (range 10 to 180). Recurrent stricture occurred in 4 men with prior urethral manipulation and it was managed successfully by direct vision internal urethrotomy alone.

Conclusions: After blunt straddle injury to the perineum the primary morbidity is anterior urethral stricture, for which suprapubic cystostomy is appropriate initial management. The majority of patients require surgery but with careful preoperative planning and adequate resection of fibrotic tissue the long-term success rate can approach 95\%. If it arises, recurrent stricture responds well to direct vision internal urethrotomy alone.

doi: $10.1590 / S 1677-553820100001000021$

Management of low velocity gunshot wounds to the anterior urethra: the role of primary repair versus urinary diversion alone

Husmann DA, Boone TB, Wilson WT

Department of Surgery, University of Texas Southwestern Medical Center, Dallas

J Urol. 1993; 150: 70-2

The management of partial transection of the anterior urethra following penetrating penile injuries is controversial. Optional therapeutic techniques range from a primary sutured reapproximation to urinary diversion alone. We recently managed 17 low velocity gunshot wounds to the external genitalia in which the missile traversed the penile corpus cavernosum, and was associated with less than $40 \%$ transection of the corpus spongiosum and anterior urethra. Nine patients were managed with suprapubic diversion, skin débridement and corporeal 
closure along with placement of a urethral catheter. Eight patients were managed by suprapubic diversion, débridement, closure of the corporeal bodies and a primary sutured reapproximation of the anterior urethra. Urethral strictures developed in 7 patients (78\%) managed by a suprapubic tube and urethral stenting during an average followup of 20 months (range 18 to 24). In contrast, 1 patient (12\%) managed by a sutured urethral approximation had a urethral stricture during an average followup of 20 months (range 18 to $30, \mathrm{p}<0.01$ ). Our data support a significantly better prognosis for partial transection of the anterior urethra secondary to low velocity gunshot wounds if managed by aggressive wound débridement, corporeal repair, placement of a suprapubic catheter and primary repair of the urethra.

doi: 10.1590/S1677-553820100001000022 